\title{
Applied and Theoretical Research on the Parameter-Dependent Efficiency of a Coilgun
}

\author{
Alexander Asriyan, \\ Physics and Mathematics Specialized School named after Artashes \\ Shahinyan, under the Yerevan State University, Yerevan, Armenia
}

Doi: 10.19044/esj.2019.v15n12p129 URL:http://dx.doi.org/10.19044/esj.2019.v15n12p129

\begin{abstract}
This research paper focuses on determining the dependence of a coilgun's efficiency on its working parameters. Due to its working principal, the linear acceleration process of a ferromagnetic projectile can be described as a "shot". The ratio of the projectile's kinetic energy to the potential energy stored in the capacitors was used to calculate the efficiency of each shot. The equation of force pushing the projectile was derived theoretically. For health and safety reasons, measurements were taken with projectile speeds under $15 \mathrm{~m} / \mathrm{s}$. A kinematic method was used to measure the speeds of the projectiles. Iron was chosen as the projectile material due to its ferromagnetic properties. After understanding the major factors that affect the coilgun's efficiency and by optimizing them, an efficiency value of $6.7 \%$ was achieved.
\end{abstract}

Keywords: Coilgun, efficiency, electromagnetic accelerator, working parameters

\section{Introduction}

A coilgun is a type of electromagnetic accelerator that accelerates a ferromagnetic projectile by means of creating short-term high-density magnetic flux in the tubular open chamber where the projectile is stored. The ferromagnetic projectile's geometrical shape is cylindrical. Eddy currents appear on the side surface of the projectile. Therefore, the radial component of the magnetic field pushes the projectile along the barrel by means of Ampere's force. This type of coilgun consists of an induction coil wrapped around a tube made from a dielectric material that acts like a barrel for linear acceleration. The high current pulse that is needed for creating strong magnetic fields inside the barrel is achieved by using capacitors. The practical use of these types of accelerators is limited[Kaye et al., 2002] due to their extremely low efficiency, close to $1 \%$ [Barros et al., 2003]. No well-known practical research has been conducted to understand the problems hindering 
the achievement of higher values of efficiency. The maximum achieved efficiency of this type of electromagnetic accelerator is about 7\% [Wikipedia, "Coilgun"]. The following sections consist mainly of applied research followed by an additional theoretical analysis. Permissible approximations were applied in both parts of the research, such as air resistance and the induction coil's length [Tao et al., 2015].

\section{Experimental Setup}

\section{Experimental Apparatus}

Due to the low velocities of the projectile, a kinematic method was used to measure the initial velocity values. The kinematic method consists of measuring the horizontal range of the projectile after leaving the barrel and the free fall time from the coilgun's barrel to the horizontal floor. Since the module of the horizontal component of the velocity was obtained from the ratio of the measured length to the free fall time, the coilgun's firing direction was set up parallel to the floor. This was done so that the absolute velocity module would be equal to the horizontal component of the velocity. Aluminum foil was spread on the floor in the firing direction so it would be easier to mark the location of the projectiles on the floor. The observation error of the measured horizontal range length is $0.5 \mathrm{~mm}$. The capacitor bank voltage readings were also taken by using a standard multimeter, and the observation error of the multimeter readings is $50 \mathrm{mV}$.

\section{Test Specimens}

The barrel of the coilgun used in this research was made from a plastic tube with a diameter of $8 / 1 \mathrm{~mm}(8 \mathrm{~mm}$ outer diameter and $1 \mathrm{~mm}$ wall thickness) and a length of $118 \mathrm{~mm}$. The induction coil's measured inductance is $22.4 \mathrm{mH}$, the number of turns is 424 , and the diameter is $32 / 12 \mathrm{~mm}$. The wire used in the inductance coil is enameled copper wire, with a cross-section diameter of $0.72 \mathrm{~mm}$ and the measured total resistance of the wire is $5.6 \Omega$. The projectiles were made by cutting sections of iron rods with diameters of $4 \mathrm{~mm}$ and $6 \mathrm{~mm}$, and the lengths of the projectiles differ from $10 \mathrm{~mm}$ to $60 \mathrm{~mm}$. The capacitors bank capacity values used during this research differ from $220 \mu \mathrm{F}$ to $1998 \mathrm{uF}$. The shots were taken with capacitor voltage values between $60 \mathrm{~V}$ and $180 \mathrm{~V}$.

\section{Experimental Procedure}

The method used to measure the velocity required the coilgun to be positioned parallel to the floor. Before conducting each experiment, the coilgun barrel's height from the floor was measured. The free-fall time corresponding to that height was also calculated which is the time period between the projectile leaving the coilgun's barrel and the projectile hitting 
the floor. That time period is marked as $t_{0}$. $t_{0}$ is constant during each experiment. After each shot, the range of the projectile was measured and divided by $t_{0}$, thus getting the initial velocity of the projectile. By having such parameters as capacitor bank's voltage reading and capacity, and projectile's mass and initial velocity, capacitor bank's potential and projectile's kinetic energies were calculated according to each shot. Thus, the ratio of projectile's kinetic energy and capacitor bank's potential energy equals to the efficiency of each shot. During each experiment, one of the parameters that were given to the coilgun (capacitor bank's voltage, projectile position in the barrel [Perotoni et al., 2017], capacitor bank's capacity, projectile's mass and dimensions) was variable throughout the experiment to understand how the efficiency of the coilgun depends on that specific parameter. The value of the variable at which maximal efficiency was achieved was taken into consideration during the following experiments. During the first experiment, the dependency of the coilgun's efficiency on the capacitor's voltage was determined. In the next experiment, the value of the capacitor's voltage remained $60 \mathrm{~V}$ during each shot but the projectile's position was variable and marked as $\mathrm{X}$ (the distance between the back edge of the barrel and the back edge of the projectile). In the theoretical analysis section, the position variable can be marked as $\mathrm{z}$ (the distance between the center of the inductance coil and the front edge of the projectile). The dependency of the coilgun's efficiency on capacitor's capacity was also tested. In the following experiments, the mass and linear dimensions of the projectiles were changed in order to achieve the highest possible efficiency. The voltage and capacity of the capacitor bank remained constant during each experiment. The geometrical shape of the projectiles was chosen to be cylindrical.

\section{Results}

\section{Capacitor's Voltage}

A series of shots were fired with capacitor voltages ranging from $60 \mathrm{~V}$ to $170 \mathrm{~V}$. The following results are shown in Figure 2. It can be seen from the graph that the efficiency value fluctuates around $1 \%$. This result was expected and it proves that the dependence of a coilgun's efficiency on the capacitor's voltage value is negligible and the change in efficiency can be disregarded.

\section{Projectile's Position}

The projectile's position in the coilgun's barrel is the distance between the back edge of the projectile and the back edge of the barrel, and it is marked as X. By changing its value before each shot, the dependency of the coilgun's efficiency on the projectile's position can be obtained. In Figure 3, the back edge of the barrel is denoted by the axis value of 0 . Therefore, it can be concluded that the coilgun's efficiency definitely depends on the projectile's 
position in the barrel. The maximum value of efficiency is achieved at $\mathrm{X}=$ $4 \mathrm{~mm}(\mathrm{z}=10 \mathrm{~mm})$ and is equal to $3.83 \%$.

(A)

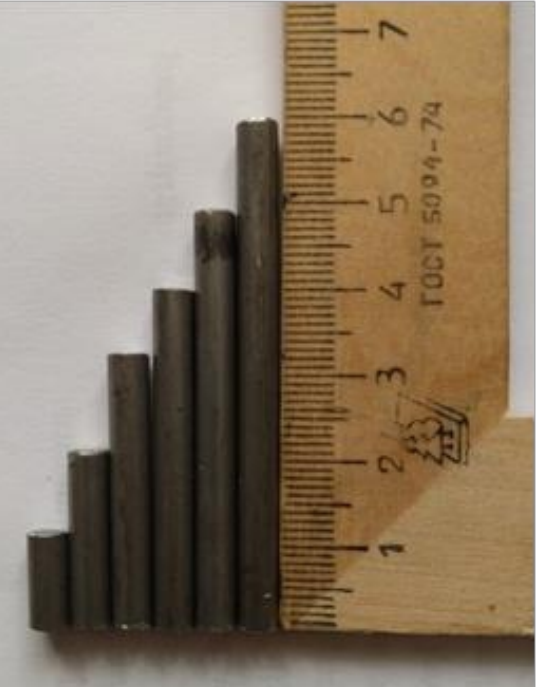

Figure 1. Tested projectile specimens made from iron rods with cross-section diameters of $4 \mathrm{~mm}$

\section{Capacitor's Capacity}

Capacity values from $220 \mu \mathrm{F}$ to $1998 \mu \mathrm{F}$ were tested to determine the most efficient combination. The results can be seen in Figure 4. A significant dependence of the coilgun's efficiency on this factor was also expected and now it is proven experimentally. Maximum efficiency is obtained at $880 \mu \mathrm{F}$ and equals $2.48 \%$.

\section{Weight and Dimensions of the Projectile}

From an iron rod with a cross-section diameter of $4 \mathrm{~mm}, 6$ cylindrical sections [Bresie et al., 1995] were made with lengths ranging from 10-60mm (Figure 1A). The distance between the front side of the projectile and the center of the inductance coil(s) remained constant during all shots. The dependence of the coilgun's efficiency on the projectile's mass is shown in Figure 5. The maximum value was achieved with the $50 \mathrm{~mm}$ long projectile, with a weight of $7.55 \mathrm{~g}$, and is equal to $3.12 \%$. In the next step of this experiment, an iron rod with a cross-section diameter of $6 \mathrm{~mm}$ was used to make new projectiles with 3 different lengths $-34 \mathrm{~mm}, 40 \mathrm{~mm}$, and $50 \mathrm{~mm}$ (Figure 1B). The $34 \mathrm{~mm}$ length was chosen specifically to replicate the weight of the projectile with a $4 \mathrm{~mm}$ cross-section diameter and a length of $50 \mathrm{~mm}$. The results are shown in Table 1 . The efficiency of the $40 \mathrm{~mm}$ projectile with a weight of $8.43 \mathrm{~g}$ is $5.18 \%$. The dependency of the coilgun's efficiency on 
the projectile's position was obtained again specifically for the projectile with a cross section of $6 \mathrm{~mm}$ and a weight of $8.43 \mathrm{~g}$ to optimize the efficiency. The results are shown in Figure 6. The maximum efficiency is achieved at $\mathrm{X}=$ $0.8 \mathrm{~mm}$ and is equal to $6.65 \%$.

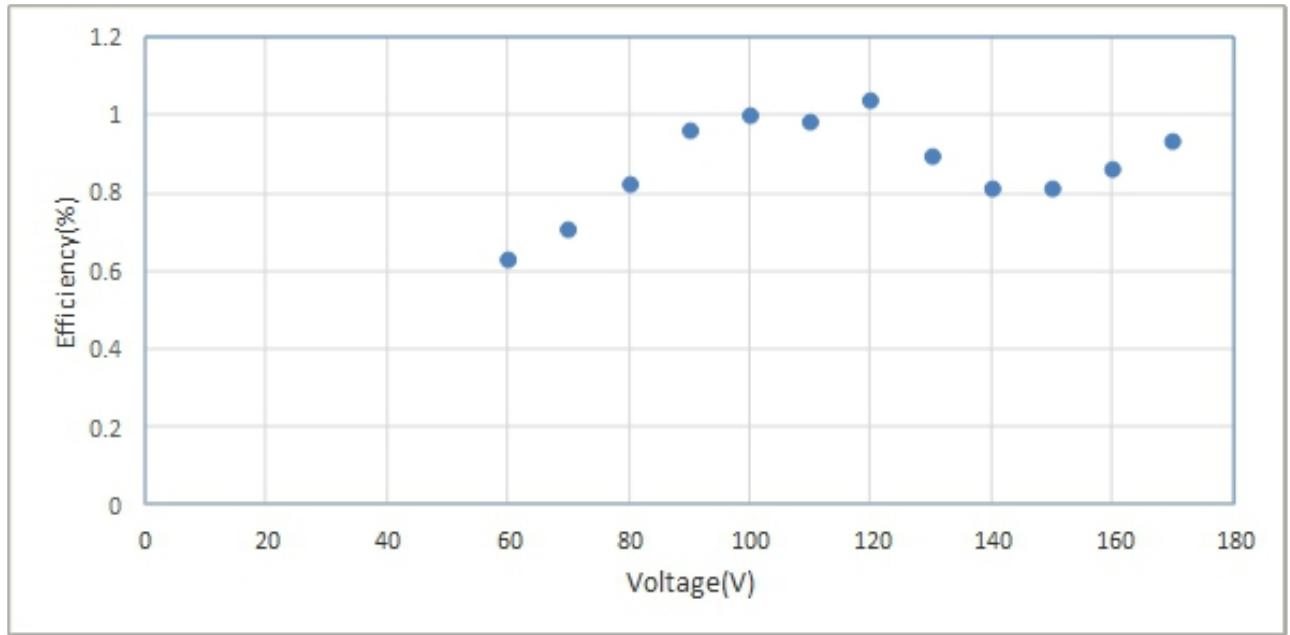

Figure 2. Coilgun Efficiency on capacitor bank's voltage

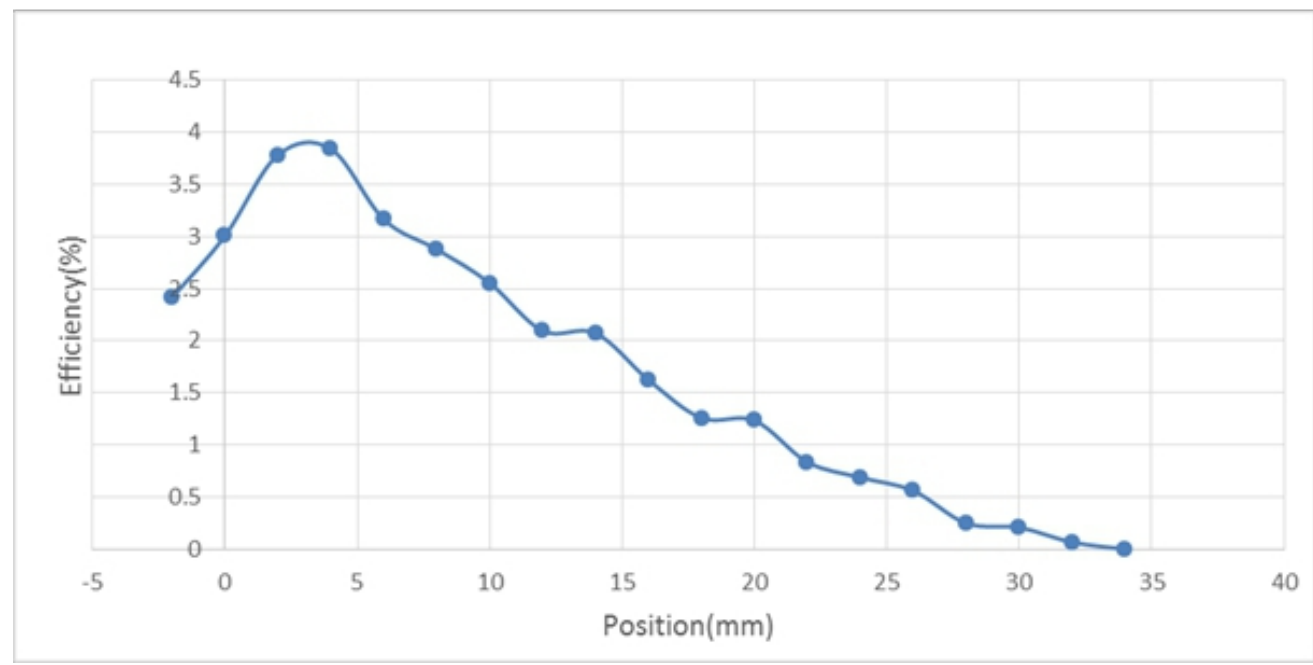

Figure 3. Coilgun Efficiency on projectile position 


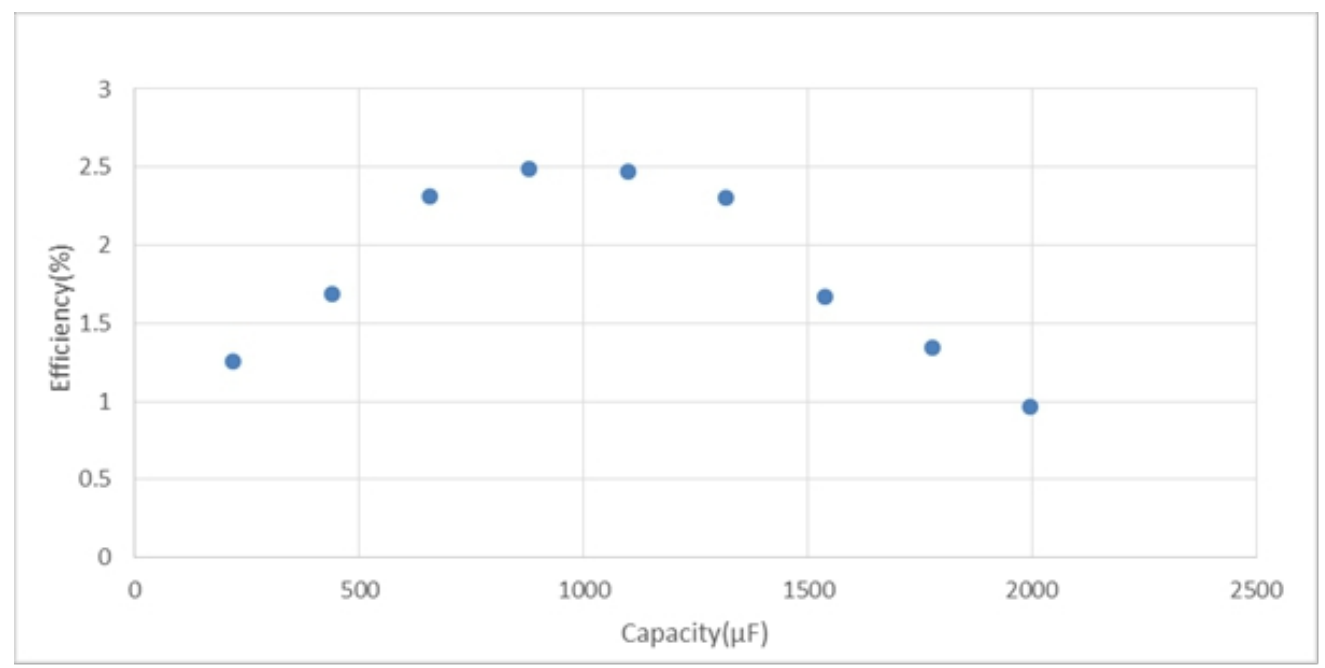

Figure 4. Coilgun Efficiency on capacitor banks's capacity

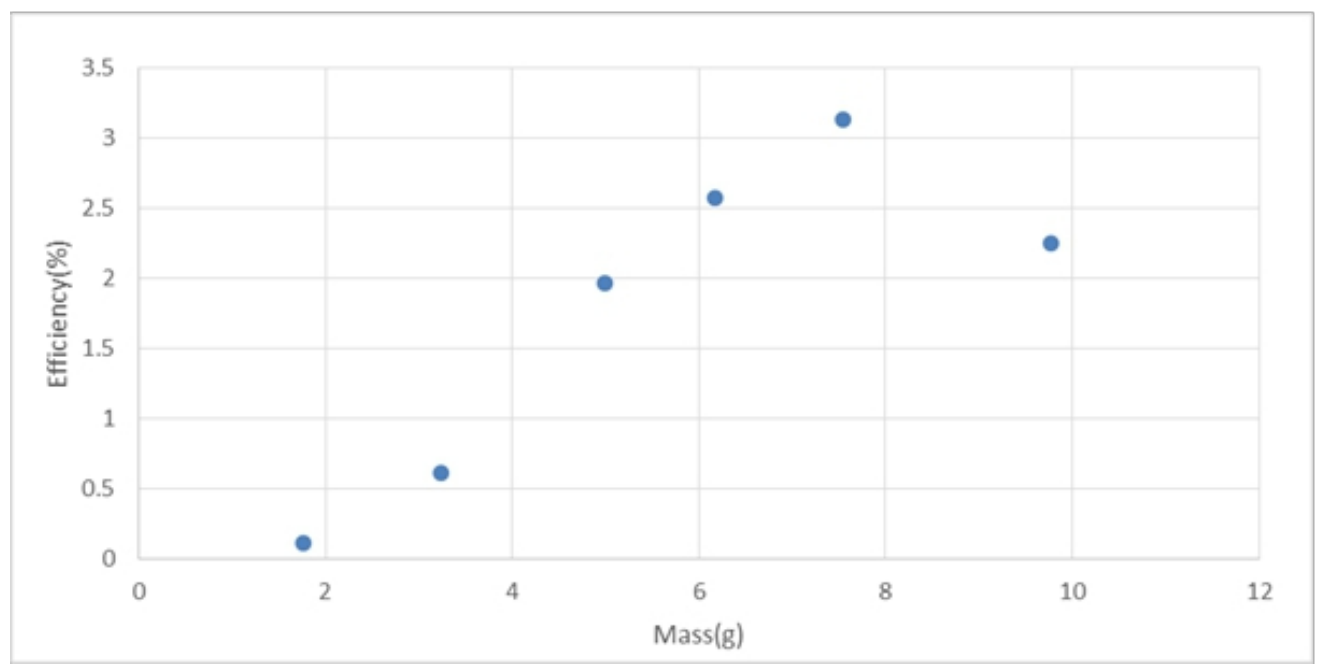

Figure 5. Coilgun Efficiency on projectile mass

Table 1. Results of the testing of the projectiles with cross-section diameter of $6 \mathrm{~mm}$

\begin{tabular}{ccccc}
\hline $\begin{array}{c}\text { Projectile } \\
\text { weight }(\mathrm{g})\end{array}$ & $\begin{array}{c}\text { Projectile length } \\
(\mathrm{mm})\end{array}$ & $\begin{array}{c}\text { Horizontal range } \\
(\mathrm{m})\end{array}$ & $\begin{array}{c}\text { Initial velocity } \\
(\mathrm{m} / \mathrm{s})\end{array}$ & $\begin{array}{c}\text { Efficiency } \\
(\%)\end{array}$ \\
10.6 & 50 & 1,21 & 11,16 & 4.63 \\
8.43 & 40 & 1,43 & 13,26 & 5.18 \\
7.06 & 34 & 1,31 & 12,11 & 3.63 \\
\hline
\end{tabular}




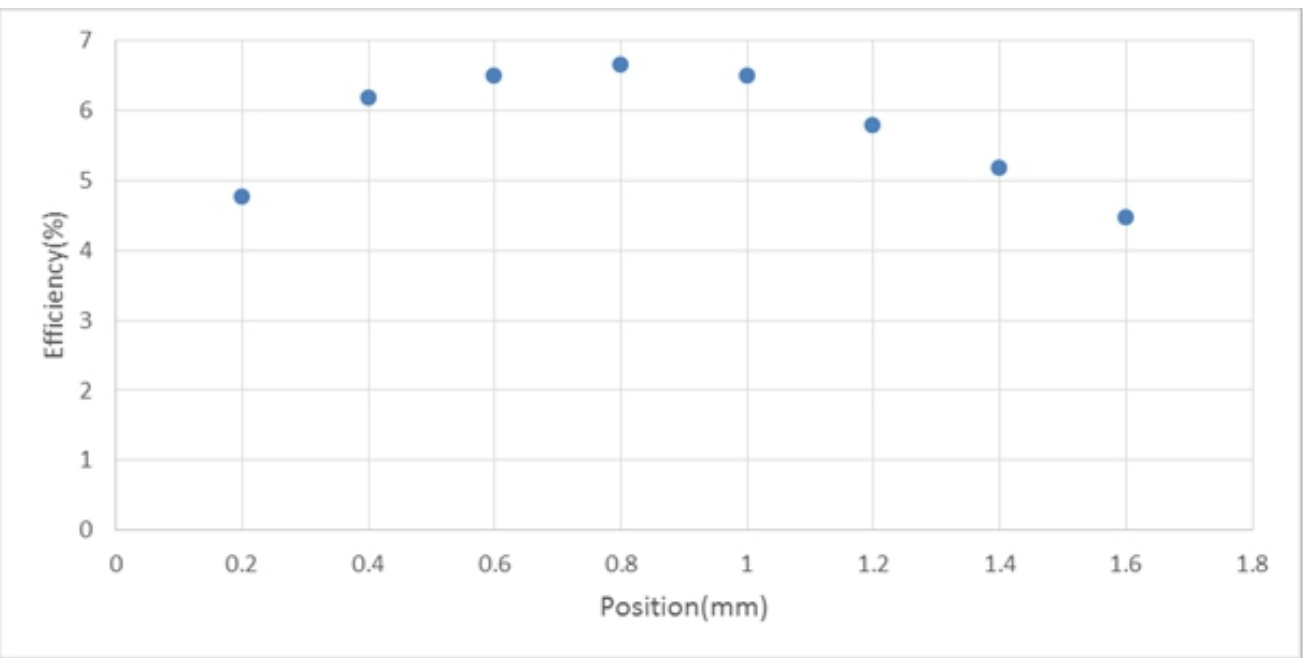

Figure 6. Coilgun Efficiency on projectile position with the projectile with cross-section diameter of $6 \mathrm{~mm}$ and length of $40 \mathrm{~mm}$

\section{Theoretical Addition}

In this simplified model of a coilgun [Slabodyanyuk et al., 2012] the coil is replaced by a single loop with a radius of $\mathrm{R}$ and the inductance is neglected. The projectile has a cylindrical shape with a radius of $r$ and a length of $L$ made of ferromagnetic material with $\mu$ permeability. Let's assume that the current in the loop is and the projectile is $\mathrm{z}$ distance away from the center of the loop in the direction of its axis perpendicular to the surface of the loop. The module of the magnetic field in the direction of its axis at a distance of $\mathrm{z}$ from the loop can be found by Equation 1 .

$$
B_{z}=\frac{\mu_{0} I_{0}}{2} \cdot \frac{R^{2}}{\left(R^{2}+z^{2}\right)^{\frac{3}{2}}}
$$

As the axisymmetric magnetic field changes along the axes, a radial component is observed. From Gauss' law of magnetism, total magnetic flux through a closed surface is equal to zero. Let consider a thin axisymmetric cylinder with a height of $\mathrm{dz}$ and radius of $\mathrm{r}$. The lower edge of the cylinder is $\mathrm{z}$ distance away from the loop. The magnetic fluxes through the lower edge, side surface and upper edge are $\Phi_{1}, \Phi_{2}$ and $\Phi_{3}$ respectively.

$$
\begin{gathered}
\Phi_{1}=-\mathrm{B}(\mathrm{z}) \cdot \pi \mathrm{r}^{2} ; \\
\Phi_{2}=\mathrm{B} \cdot 2 \pi \mathrm{rdz} ; \\
\Phi_{3}=\mathrm{B}(\mathrm{z}+\mathrm{dz}) \cdot \pi \mathrm{r}^{2} ;
\end{gathered}
$$




$$
\begin{gathered}
\Phi_{1}+\Phi_{2}+\Phi_{3}=0 ; \\
-B(z) \cdot \pi r^{2}+B_{r} \cdot 2 \pi r d z+B(z+d z) \cdot \pi r^{2}=0 ;
\end{gathered}
$$

By solving Equation 6, $\mathrm{B}_{\mathrm{r}}$ is equal to:

$$
B_{r}=\frac{r}{2} \cdot \frac{B(z)-B(z+d z)}{d z}=-\frac{r}{2} \cdot \frac{d B(z)}{d z}=-\frac{r}{2} \cdot \frac{d}{d z}\left(\frac{\mu_{0} I_{0}}{2} \cdot \frac{R^{2}}{\left(R^{2}+z^{2}\right)^{\frac{3}{2}}}\right)=\frac{3 \mu_{0} I_{0} R^{2} r}{4} \frac{z}{\left(R^{2}+z^{2}\right)^{\frac{5}{2}}} ;
$$

To find the Eddy currents induced on the projectile's side surface, let consider the projectile as a solenoid with a length of $L$, a cross-section radius of $r$ $(\mathrm{r}<<\mathrm{L})$, and a single loop. Iron's magnetic permeability is $\mu$. From Ampere's circuital law:

$$
\mu_{0} \mathrm{i}=\mu \mathrm{B}_{\mathrm{z}} \mathrm{L} ;
$$

From Equation 8, the induced current on the projectile surface is equal to:

$$
\mathrm{i}=\frac{\mu \mathrm{B}_{2} \mathrm{~L}}{\mu_{0}} ;
$$

Ampere's force equation for the projectile is written in Equation 10.

$$
F=2 \pi r i B_{r}=\frac{3 \pi \mu_{0} l_{0} R^{2} r^{2} i}{2} \cdot \frac{z}{\left(R^{2}+z^{2}\right)^{\frac{5}{2}}}=\frac{3 \pi \mu \mu_{0} l_{0}^{2} r^{2} R^{2} L}{4} \cdot \frac{z}{\left(R^{2}+z^{2}\right)^{\frac{5}{2}}} \cdot \frac{R^{2}}{\left(R^{2}+z^{2}\right)^{\frac{3}{2}}}=\frac{3 \pi \mu \mu_{0}{ }_{0}^{2} r^{2} L}{4 R^{3}} \cdot \frac{\frac{z}{R}}{\left(1+\frac{z^{2}}{R^{2}}\right)^{4}} ;
$$

For ease of plotting, let define $\mathrm{F}_{0}$ and $\mathrm{x}$ :

$$
\begin{gathered}
F_{0}=\frac{3 \pi \mu \mu_{0} 0_{0}^{2} r^{2} L}{4 R^{3}} \\
x=\frac{z}{R^{\prime}}
\end{gathered}
$$

The new function is:

$$
f(x)=\frac{F}{F_{0}}=\frac{x}{\left(1+x^{2}\right)^{4}}
$$

The plot of Function $\mathrm{f}(\mathrm{x})$ in equation 13 is shown in Figure 7. Now, let find the derivative of Function 13 and equalize it to zero.

$$
\frac{d}{d x}(f(x))=\frac{d}{d x}\left(\frac{x}{\left(1+x^{2}\right)^{4}}\right)=\frac{\left(1+x^{2}\right)^{4}-x \cdot 4\left(1+x^{2}\right)^{3} \cdot 2 x}{\left(1+x^{2}\right)^{4}}=\frac{1-7 x^{2}}{1+x^{2}}=0 ;
$$


By solving Equation 14, the maximum value of Function $\mathrm{f}(\mathrm{x})$ in equation 13 is at $\mathrm{x}=$

$$
\frac{1}{\sqrt{7}} \approx 0.377
$$

Therefore, the function maximum is equal to:

$$
f(x)_{\max }=\frac{\frac{1}{\sqrt{7}}}{\left(1+\left(\frac{1}{\sqrt{7}}\right)^{2}\right)} \approx 0.22
$$

And the maximal force pushing the projectile can be calculated from Equation 16.

$\mathrm{F}_{\max }=0.22 \mathrm{~F}_{0} \approx 0.166 \cdot \frac{\pi \mu \mu_{0} I_{0}^{2} \mathrm{r}^{2} \mathrm{~L}}{\mathrm{R}^{3}}$

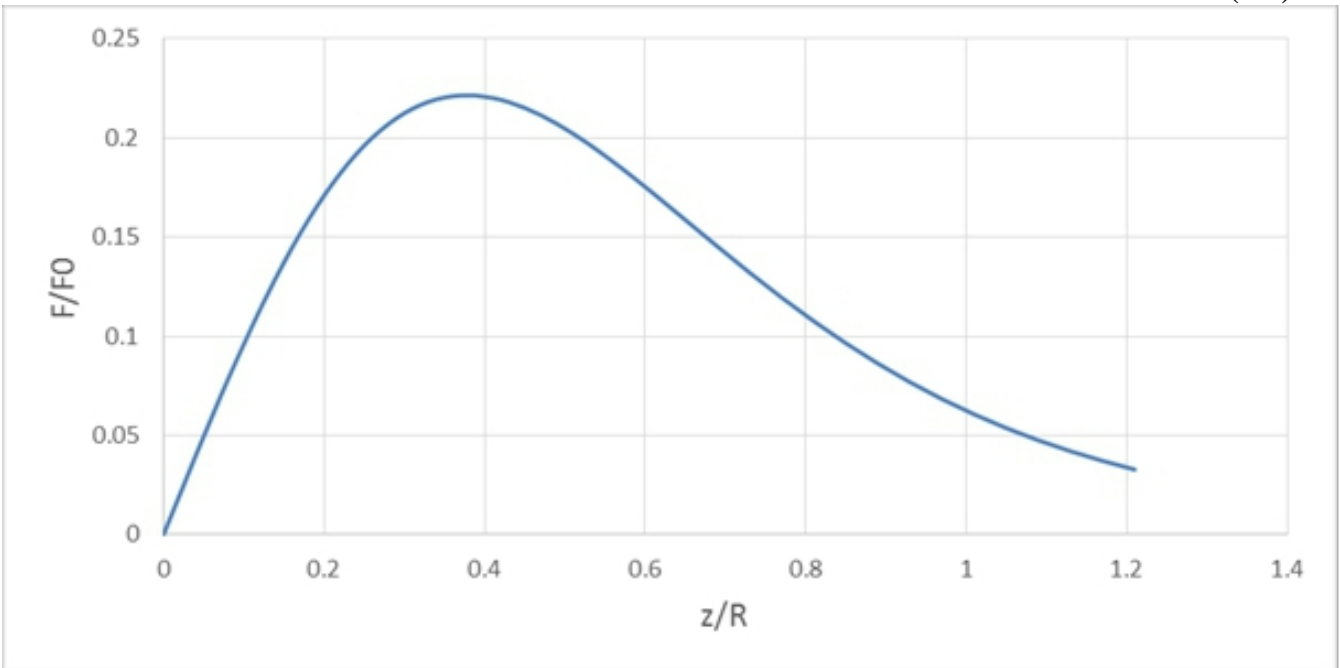

Figure 7. The Graph of the Function $\mathrm{f}(\mathrm{x})$

\section{Conclusion and Summary}

After testing the dependence of a coilgun's efficiency on the working parameters, such as the capacitor bank's voltage, projectile position in the barrel, capacitor bank's capacity, and projectile's mass and dimensions, the following conclusions can be summarized:

Capacitor's bank voltage change affected the coligun's efficiency insignificantly. As a result of this, the efficiency fluctuated around 1\%. A major change in efficiency of the coilgun was observed when the projectile's position was varied during the experiment. By applying the most efficient value of the projectile's position in the barrel, the coilgun's efficiency was 
increased up to $3.83 \%$. Capacitor bank's capacity change also affected the efficiency of the coilgun in a significant way. From the tested specimens, the most efficiency was gained with capacitor bank's capacity of $880 \mu \mathrm{F}$ and was equal to $2.48 \%$. $4 \mathrm{~mm}$ cross-section diameter cylindrical projectiles with lengths of $10 \mathrm{~mm}$ to $60 \mathrm{~mm}$ and $6 \mathrm{~mm}$ cross-section diameter ones with lengths of $34 \mathrm{~mm}, 40 \mathrm{~mm}, 50 \mathrm{~mm}$ was tested. With the $6 \mathrm{~mm}$ cross-section diameter projectile with length of $40 \mathrm{~mm}$, the maximal efficiency was achieved and it was equal to $5.18 \%$. After optimizing the projectile's position for the $6 \mathrm{~mm}$ cross section diameter projectile with length of $40 \mathrm{~mm}$, the maximal efficiency of $6.68 \%$ was achieved, which is the highest value registered during the whole research.

Thus, Coilgun's efficiency significantly depends on the following parameters; projectile's position in the barrel, capacitor's capacity, and weight and dimensions of the projectile. After optimizing these parameters, efficiency value of $6.68 \%$ was achieved. Force equation was derived theoretically and this determines the ways of further analysis and improvement of the efficiency of a coilgun.

\section{References:}

1. Kaye, R. J., Turman, B.N. \& Shope, S.L., Conference Record of the Twenty-Fifth International Power Modulator Symposium, 2002 and 2002 High-Voltage Workshop, Applications of coilgun electromagnetic propulsion technology. https://ieeexplore.ieee.org/document/1189573/keywords\#keywords

2. Bresie, D.A., Bacon, J.L., Ingram S.K., Kennington, K.S., Weeks D.A., IEEE Transactions on Magnetics ( Volume: 31 , Issue: 1, Jan. 1995 ), SPEAR coilgun. https://ieeexplore.ieee.org/document/364645

3. Perotoni, M. B., Mergl, M., Bernardes, V.A., IEEE Transactions on Plasma Science (Volume: 45, Issue: 6 , June 2017 ), Coilgun Velocity Optimization With Current Switch Circuit. https://ieeexplore.ieee.org/document/7927775

4. Tao, X., Wang, S., Huangfu, Y., Wang, S., Wang, Y., IEEE Transactions on Plasma Science (Volume: 43 , Issue: 5 , May 2015 ), Geometry and Power Optimization of Coilgun Based on Adaptive Genetic Algorithms.https://ieeexplore.ieee.org/document/7061927

5. Barros, S., Sam Barros PowerLabs, PowerLabs Single Stage Coilgun Research 2.0 http://www.powerlabs.org/coilgun.htm

6. Slabodyanyuk, A. I., Mishuk, A. A., Anculevich, V. I., Markovich L.G.,(2012), Theoretic Tour of 2012 Belarusian Republican Olympiad, Problem 11-1 "Railgun".

http://www.belpho.org/problems/third/3_etap_2012_theoretical_solut ions.pdf 